\section{IMPRESSUM}

\section{HERAUSGEBER UND VERLAG:}

Expert Fachmedien GmbH

Aachener Str. 172, 40223 Düsseldorf

Deutschland

Telefon: +49 (0) 211 / 15 91-2 10, Fax: +49 (0) 2 11 / 15 91-1 50

www.expert-fachmedien.de, www.keramische-zeitschrift.info

Geschäftsführer: Paul Robert Hoene

Inhaber der Titelrechte: DVS Media GmbH, Düsseldorf, Deutschland

\section{REDAKTION:}

Dr. Hubert Pelc (Leitung, V.i.S.d.P.)

Telefon: +49 (0) 211 / 15 91-2 30 // E-Mail: hubert.pelc@dvs-hg.de

Dr.-Ing. Paul-Eberhard Keilbar

Telefon: +49 (0) 341 / 9410237 // E-Mail: keilbar@t-online.de

Sylvia Hanagan (Redaktionsassistenz)

Telefon: +49 (0) 2 11 / 15 91-2 31 // E-Mail: sylvia.hanagan@dvs-hg.de

\section{REDAKTIONSKOMITEE:}

Vorsitzender: G. Klein (Deutschland)

H. Brückner (Österreich), D. R. Dinger (USA), F. Kerbe (Deutschland),

0 . Krause (Deutschland), W. Kollenberg (Deutschland), J. H. Mendheim (Deutschland),

H. Mörtel (Deutschland), A. Ravaglioli (Italien), M. Röhrs (Deutschland), G. Routschka (Deutschland), L. Schyia (Deutschland), J. Werner (Deutschland)

\section{LAYOUT:}

Julia Bobe, Expert Fachmedien GmbH

DRUCK:

D+L Printpartner GmbH

Schlavenhorst 10, 46395 Bocholt

\section{ANZEIGEN:}

Christiane Czech (Leitung)

Telefon: +49 (0) 211 / 15 91-1 57, E-Mail: christiane.czech@dvs-hg.de

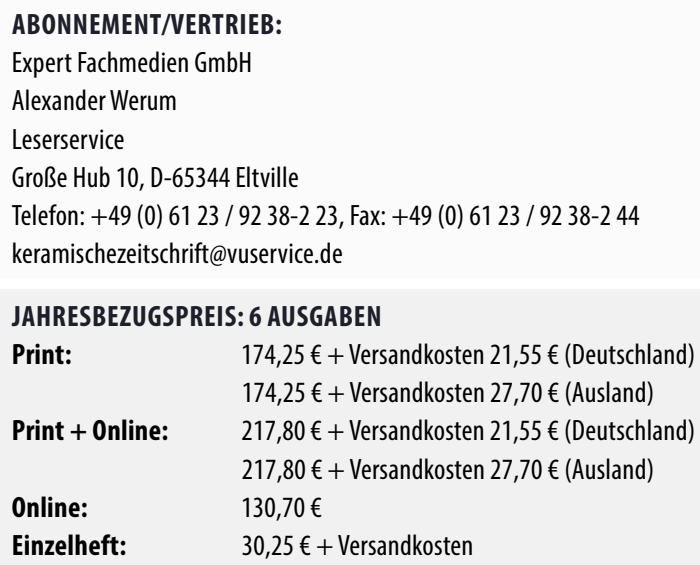

\section{JAHRESBEZUGSPREIS: 6 AUSGABEN}

Print: $\quad 174,25 €+$ Versandkosten $21,55 €$ (Deutschland) $174,25 €+$ Versandkosten 27,70€ (Ausland)

Print + Online: $\quad 217,80 €+$ Versandkosten $21,55 €$ (Deutschland)

$217,80 €+$ Versandkosten $27,70 €$ (Ausland)

Online: $\quad 130,70 €$

Einzelheft: $\quad 30,25 €+$ Versandkosten

๑) Copyright 2016 // Expert Fachmedien GmbH // D-40223 Düsseldorf, Deutschland

\section{ISSN: 0023-0561}

Die „KERAMISCHE ZEITSCHRIFT“ sowie alle in dieser Zeitschrift enthaltenen Beiträge, Bilder und Tabellen sind urheberrechtlich geschützt. Mit Ausnahme der gesetzlich zugelassenen Fälle ist eine Verwertung ohne Einwilligung der Expert Fachmedien GmbH strafbar. Für unverlangt eingesandte Manus kripte wird keine Haftung übernommen.

Mit der Annahme zur Veröffentlichung überträgt der Autor dem Verlag das ausschließliche Verlagsrecht für die Zeit bis zum Ablauf des Urheberrechts. Diese Rechteübertragung bezieht sich insbesondere auf das Recht des Verlages, das Werk zu gewerblichen Zwecken per Kopie (Mikrofilm, Fotokopie, CD-ROM oder andere Verfahren) zu vervielfältigen und/ oder in elektronische oder andere Datenbanken aufzunehmen.

\title{
Blickpunkt Pulvermetallurgie
}

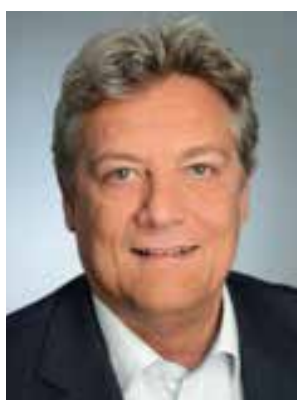

Liebe Leserinnen und Leser,

Nachdem unser Sonderheft zum letztjährigen Hagener Pulvermetallurgie-Symposium erfreulicherweise gut angenommen wurde, widmen wir uns mit der vorliegenden Ausgabe auch dieses Jahr wiederum schwerpunktmäßig der Pulvermetallurgie. Wie der Schriftzug „Blickpunkt Pulvermetallurgie" auf der Titelseite erahnen lässt, werden wir von nun an insbesondere die Hagener Veranstaltung und den FPM - Fachverband Pulvermetallurgie (Organisator des Pulvermetallurgie-Symposiums) sowie seine Mitgliedsfirmen dauerhaft publikationstechnisch begleiten.

Das diesjährige, mittlerweile 35. Hagener Symposium am 24. und 25. November steht unter dem übergeordneten Leitthema „Zerspanung von und mit pulvermetallurgischen Werkstoffen" und verknüpft vielseitige Inhalte aus den Bereichen der Pulvermetallurgie und Zerspanung. Pulvermetallurgische Werkstoffe bilden häufig das Ausgangsmaterial für Zerspanungswerkzeuge. Andererseits besteht die Herausforderung darin, pulvermetallurgisch hergestellte Werkstoffe verschiedener Art anforderungsgerecht und effektiv zu zerspanen. Zahlreiche namhafte Referenten werden abwechslungsreiche Vorträge zu aktuellen Themen sowohl aus dem Bereich der Wissenschaft als auch aus dem industriellen Umfeld präsentieren. In den Fachvorträgen werden schwerpunktmäßig die Themengebiete Herstellung und Optimierung innovativer Zerspanungswerkzeuge sowie die Zerspanung pulvermetallurgischer Werkstoffe und Sonderwerkstoffe behandelt.

Durch die Kombination der beiden Disziplinen „Pulvermetallurgie“ und „Zerspanung" bildet die diesjährige Veranstaltung somit eine einmalige Gelegenheit, um den interdisziplinären Austausch zwischen den eng verbundenen Fachgebieten zu intensivieren und ermöglicht es, ein breit gefächertes Themenspektrum abzudecken. Das Symposium wird wiederum von einer Fachausstellung mit ca. 60 Ausstellern begleitet, die einen idealen Rahmen für vertiefende Fachgespräche bietet.

Ich würde mich natürlich sehr freuen, wenn unsere Sonderausgabe „Blickpunkt Pulvermetallurgie" bei Ihnen auch in Zukunft Anklang finden wird.

Im Namen der gesamten Redaktion wünsche ich allen unseren Lesern, Autoren und Anzeigenkunden schon jetzt eine schöne Vorweihnachtszeit, frohe Weihnachtstage und einen guten Start ins neue Jahr.
Hubert Pelc

redaktion-keramik@dvs-hg.de 